\title{
Article
}

\section{Imaging the Atomic Position of Light Cations in a Porous Network and the Europium(III) Ion Exchange Capability by Aberration-Corrected Electron Microscopy}

Mayoral, Alvaro, Hall, Reece Michael, Jackowska, Roksana and Readman, Jennifer Elizabeth

Available at http://clok.uclan.ac.uk/16506/

Mayoral, Alvaro, Hall, Reece Michael, Jackowska, Roksana and Readman, Jennifer Elizabeth ORCID: 0000-0002-8170-5533 (2016) Imaging the Atomic Position of Light Cations in a Porous Network and the Europium(III) Ion Exchange Capability by Aberration-Corrected Electron Microscopy. Angewandte Chemie International Edition, 128 (52). pp. 16361-16365. ISSN 1433-7851

It is advisable to refer to the publisher's version if you intend to cite from the work. http://dx.doi.org/10.1002/anie.201609094

For more information about UCLan's research in this area go to http://www.uclan.ac.uk/researchgroups/ and search for <name of research Group>.

For information about Research generally at UCLan please go to http://www.uclan.ac.uk/research/

All outputs in CLoK are protected by Intellectual Property Rights law, including Copyright law. Copyright, IPR and Moral Rights for the works on this site are retained by the individual authors and/or other copyright owners. Terms and conditions for use of this material are defined in the policies page. 


\title{
Imaging the atomic position of light cations in a porous network and the $\mathrm{Eu}^{3+}$ ion exchange capability by aberration corrected electron microscopy.
}

\author{
Alvaro Mayoral, ${ }^{*[a]}$ Reece M. Hall, ${ }^{[b]}$ Roksana Jackowska, ${ }^{[b]}$ and Jennifer E. Readman*[b] \\ Abstract: In the present work, ETS-10 microporous titanosilicate has been synthesized and its structure characterized by means of powder XRD and \\ aberration corrected scanning transmission electron microscopy $\left(\mathrm{C}_{\mathrm{s}}\right.$-corrected STEM). For the first time, sodium ions have been imaged sitting inside the 7 \\ membered rings. The ion-exchanged capability has been tested by the inclusion of rare earth metals (Eu, Tb and Gd) to produce a luminescent material \\ which has been studied by atomic-resolution Cs-corrected STEM. The data produced has allowed unambiguous imaging of light atoms in a microporous \\ framework as well as determining the cationic metal positions for the first time, providing evidence of the importance of advanced electron microscopy \\ methods for the study of the local environment of metals within zeolitic supports providing unique information of both systems (guest and support) at the \\ same time.
}

ETS-10 (Engelhard titanosilicate) material is a synthetic microporous crystalline solid with pore dimensions of $4.9 \AA \times 7.6 \AA$, formed by chains of corner-sharing $\mathrm{TiO}_{6}$ octahedra and $\mathrm{SiO}_{4}$ tetrahedra linked to each other by oxygen bridges ${ }^{[1]}$. The structure contains pore systems formed by 12-membered rings (MR), $7 \mathrm{MR}$ and $3 \mathrm{MR}$ where each Ti unit introduces two negative charges which must be compensated by extra-framework cations to provide a material that is highly interesting for ion exchange, adsorption, photochemistry, membrane separation, and catalysis ${ }^{[2]}$. The particular structural features of ETS-10, which contain high degrees of disorder have proven challenging for structural elucidation ${ }^{[1 b, 3]}$. As a consequence, locating the cations ${ }^{[3-4]}$ has been problematic and theoretical studies have been devoted into the elucidation of this parameter $^{[5]}$.

The behavior and potential uses of ETS-10 are directly related to the structure, defects, electronic properties and cations incorporated and a more precise characterization is required in order to understand and exploit its properties. In this context, transmission electron microscopy has emerged as a very useful method for the observation of a large variety of porous structures ${ }^{[6]}$. $\mathrm{C}_{\mathrm{s}}$-corrected STEM in combination with a high angle annular dark field detector (HAADF) has made it possible to obtain atomic resolution data of the framework and of guest elements ${ }^{[7]}$ converting it in the most appropriate method for imaging incorporated species into the structure ${ }^{[8]}$.

In the present work we aim to shed light on the elucidation of the atomic position of light elements ( $\mathrm{Na}^{+}$) in ETS-10, by means of spherical aberration corrected STEM, through its ion exchange capability of lanthanide cations.

ETS-10 material crystallizes within the $C 2 / c$ space group with unit cell parameters $a=21.00, b=21.00, c=14.51 \AA$; $b=111.128$ and $\alpha=\gamma=90.00^{\circ}[1 \mathrm{~b}]$. Figure S1 displays the $\mathrm{C}_{\mathrm{s}}$-corrected STEM-HAADF image of ETS-10 along the [110] orientation together with the chemical and structural information of the as-synthesized material. Taking into account that the STEM-HAADF method is sensitive to the atomic number of the elements, heavier atoms scatter the electrons more strongly than lighter elements and therefore they appear brighter in the images ${ }^{[11]}$, so consequently, the identification of light cations such as $\mathrm{Na}^{+}$or $\mathrm{K}^{+}$has not yet been possible in any microporous support. Figure 1 a exhibits the $\mathrm{C}_{\mathrm{s}}$-corrected STEM-ADF (annular dark field) image where all atomic columns forming the framework can be unmistakably identified. The brightest spots correspond to $\mathrm{Ti}$ atomic columns. In this mode, the pores appear in black as there no elements present to scatter the incoming electrons. The superimposed model confirms a direct correspondence of the atomic positions between the experimental data and the theoretical model. Despite the accurate concordance between all framework atoms a faint signal can be observed in the center of the 7 membered rings (MR). In figure $1 \mathrm{~b}$ the intensity profiles are plotted along the white arrow of the figure $1 \mathrm{a}$, among the three maxima observed, the central one with the strongest signal, corresponds to the Ti columns, while two others with much lower intensity are separated from the Ti columns by $\approx 3$. $\AA$. In the initial framework model (figure S1 and 1a) the 7MR are presented as empty cavities without taking into account the cationic species. However, based on the current observations and on previous reports ${ }^{[5 a, 5 d, 5 e]}$ cations may fill the $7 \mathrm{MR}$, see figure 1c. In here, $\mathrm{Na}^{+}$cations (yellow spheres) have been introduced in the center of the $7 \mathrm{MR}$. In order to match this projected distance ( $3 \AA$ ), Na cations must be sitting in the center of the $7 \mathrm{MR}$ as described by Anderson et al. ${ }^{[5 a]}$; who placed one of the $\mathrm{Na}^{+}$(denoted as $\mathrm{Na}$ (IV), according to Anderson's nomenclature) positions within the 7-ring between two orthogonal titanium-oxygen chains; this cationic position can be defined by the atomic coordinates $x=0.50000 ; y=0.43860$

[a] Dr. A. Mayoral

Advanced Microscopy Laboratory, Nanoscience Institute of Aragon University of Zaragoza

Mariano Esquillor, Edificio I+D, 50018, Zaragoza, Spain

E-mail: amayoral@unizar.es

[b] R. M. Hall, R. Jackowska, Dr. J. E. Readman

School of Physical Sciences and Computing

University of Central Lancashire

Preston, Lancashire, UK, PR1 2HE

E-mail:JEReadman@uclan.ac.uk

Supporting information for this article is given via a link at the end of the document.((Please delete this text if not appropriate)) and $z=0.75000$ (position IV). In figure S2a, the porous framework is shown with the Na (IV) site added; now the theoretical distance between the cation and the Ti column is approximately $3.39 \AA$, slightly larger than the experimental data acquired. If instead of $\mathrm{Na}$ (IV), we introduce the equivalent positions denoted as $\mathrm{Na}$ (I) $x=$ $0.14172 ; y=0.80675$ and $z=0.80792$ and $\mathrm{Na}$ (II) $x=$ $0.38857 ; y=0.56680$ and $z=0.81253$, figure S2b, the interatomic theoretical distances between $\mathrm{Na}$ and $\mathrm{Ti}$ are $2.55 \AA$, smaller than the experimental measured. However, 
if all three atomic positions are taken into account ( $\mathrm{Na}(\mathrm{I}, \mathrm{II}$ and IV)), figure S2C, it can be observed that positions $\mathrm{Na}$ (I) and $\mathrm{Na}$ (II) are very close with respect to $\mathrm{Na}$ (IV) and not resolvable by electron microscopy. If now, the distance from the center of that $\mathrm{Na}$ unit (composed by 3 cationic positions) to the Ti columns is measured the approximate value obtained is $3.09 \AA$, very similar to the experimental data suggesting that the faint signal observed in the electron microscopy images may correspond to the three $\mathrm{Na}^{+}$cationic species coexisting together.

Based on the ${ }^{23} \mathrm{Na} 30$ MAS NMR results reported by Anderson et al. ${ }^{[5 a]}$ there should be two more cationic sites for Na occupancy, denoted $\mathrm{Na}$ (III) and $\mathrm{Na}(\mathrm{V})$, see S2. The equivalent $\mathrm{Na}$ (I) and $\mathrm{Na}$ (II) positions sitting outside the $7 \mathrm{MR}$, see figure S3, are very close to the Si columns and therefore cannot be discerned by STEM imaging since the predominant signal observed would correspond to the Si due to the light nature of the $\mathrm{Na}^{+}$cations (or more specifically to the sum of $\mathrm{Na}$ and $\mathrm{Si}$ ). Positions denoted as III and V are not observed in the experimental images, which can be attributed to either the absence of cations at those sites or that alternatively, the $\mathrm{Na}$ (III) sites are too close to the ETS-10 framework to be discernable; meanwhile for the site $V$ this can be due to its low occupancy associated to the numerous possible sites where cations can be present.

Taking these assumptions into account, multislice simulations were performed in order to corroborate the experimental observations with the theoretical data. Figure S4 shows the comparison between the experimental and simulated data corroborating the perfect agreement between both images.

In order to further investigate the initial $\mathrm{Na}^{+}$positions as well as to evaluate the ion-exchange capability of ETS-10 three different cations $\left(\mathrm{Eu}^{3+}, \mathrm{Gd}^{3+}, \mathrm{Tb}^{3+}\right)$ have been attempted to be introduced replacing the $\mathrm{Na}^{+}$and/or $\mathrm{K}^{+}$. Powder $\mathrm{X}$-ray diffraction (XRD) patterns of each material as well as the parental ETS-10 are shown in figure S5. The variations in the relative intensities of the peaks corresponding to the (2 0 -4), (1 1 -4) and (6 0 -2) reflections (at 24.55, 25.37 and 25.79 2theta respectively) suggest that the ion-exchange has been successful for each material. Accurate lattice parameters for the as-synthesized ETS-10 and the ion-exchanged ETS-10 were determined by using Pawley fits (table S1 and figure S6). The $a$ parameter for the exchanged samples increased between 0.8 and $0.9 \AA$ compared to the parent ETS-10, indicating that ion-exchange had taken place. The changes in the $b$ and $c$ parameters and the $\beta$ angle were significant, but not as large as those observed for the $a$ parameter.

The same analysis was performed for the ion exchanged materials. Eu-ETS-10 images are depicted in figure 2. Figure $2 a$ presents the low magnification micrograph with the correspondent FFT inset where no extra spots were observed and it could be also indexed assuming C2/c symmetry. From this image it can be firstly observed that the presence of double pores is not as clear as it was for the as-synthesized ETS-10 (figure S1a). Interestingly, instead of the typical "double pores" a brighter signal was observed at certain positions, attributed to the presence of the heavier Eu ${ }^{3+}$ cation. Simultaneously to this image, another micrograph was recorded obtaining a complementary annular dark field micrograph (figure $2 \mathrm{~b}$ ). In this mode of operation ${ }^{[12]}$ the resulting image is less dependent on the atomic number and more affected by diffraction phenomena. With this set up, it is therefore more complicated to observe the guest species on the supports; however, it gives a perfect image of the periodic arrangement which allows a clear visualization of the "double pores" in the same positions where the stronger signal, attributed to the $\mathrm{Eu}^{3+}$, was initially observed. In order to gain information of the ionexchange capability of ETS-10, which will influence its potential applications, atomic resolution data was acquired (figure 2c). By simple imaging comparison with the original $\mathrm{Na}^{+}$loaded ETS-10 (figure $1 \mathrm{a}$ ) it is evidenced different intensity signals, in the "butterfly units", those composed by the $\mathrm{TiO}_{6}{ }^{2-}$ surrounded by the $\mathrm{SiO}_{4}$ tetrahedra and enclosed by the $\mathrm{Ti}$ wires. The intensity profile plotted in figure $2 \mathrm{~d}$ was extracted from the area marked by the white arrow. In this case, 3 maxima are observed with higher intensity in comparison with the profile shown in figure 1a. The interatomic distances measured for this case, obtained after averaged 40 distances, were shorter than the parental form, being approximately $2.59 \AA$ (in respect to the original at $3 \AA$ ). This difference suggests that the $\mathrm{Eu}^{3+}$ is only replacing certain $\mathrm{Na}+$ sites as the distance measured for the exchanged material is more in agreement with the projected $\mathrm{Na}+(\mathrm{I})-\mathrm{Na}+(\mathrm{II})-\mathrm{Ti}$ theoretical distance shown in figure S2b; making it possible to comprehend not only the ion-exchange performance of ETS-10 but also corroborate the initial Na sites. Interestingly, we have not observed any evidence of the presence of zig-zag or dimers-like geometries as it has been previously proposed for another trivalent cations ${ }^{[5 e]}$. Likewise, no signal of Eu ${ }^{3+}$ was observed within the $12 \mathrm{MR}$ that would correspond to the site (III) and/or (V).

In addition to these straightforward observable differences; the intensity recognizable in the Si columns, which are in the corner of each "butterfly" unit, is also higher in comparison to the initial material (see Fig. S7). Figure S7a corresponds to the parental material with the alkali cations present. In the proposed model (figure S2 and S3), sites (I) and (II) (for the Na ${ }^{+}$ outside the $7 \mathrm{MR}$ ) are very close to the ETS-10 skeleton and the signal obtained in those positions is associated to mainly the $\mathrm{Si}-\mathrm{Si}$ columns separated by approximately $1.44 \AA$. However, after ion exchange a much stronger signal is observed associated to $\mathrm{Eu}^{3+}$, figure S7b; the Eu-Si distances measured were $2.48 \AA$, significantly larger than those obtained for the initial ETS-10. Such variation corroborates that the first interatomic distance corresponds to two Si columns. Meanwhile, after ion exchange, $\mathrm{Eu}^{3+}$ cations occupy the $\mathrm{Na}^{+}(\mathrm{I})$ and $\mathrm{Na}^{+}$(II) sites that now appear brighter due to the higher scattering factor of $\mathrm{Eu}^{3+}$ in comparison with the framework, making able to conclude that the distance measured corresponds to the $\mathrm{Eu}^{3+}$ cations and the Si from the frameworks (see figure S8 for the schematic representation). With this method it is possible to evaluate the ion exchange performance of porous solids and also it is proven to be a good method for locating 
light elements within the framework. Image simulations were performed on ETS-10 with Eu cations in the $\mathrm{Na}$ (I) and $\mathrm{Na}$ (II) sites. Figure S9 shows the comparison between the experimental and simulated data corroborating the perfect agreement between both images.

It has been previously reported ${ }^{[1 c]}$ that the "double pores" are not completely hollow channels along the $z$ axis, (perpendicular to the paper) and it has been proved the presence of ETS-10 framework at those defects. This is corroborated when the ETS-10 is ion-exchanged with heavy elements as it is the case here. The low magnification image, figure 3a, does not show the presence of dark units (as they are clearly observable in figure S1a) which correspond to the "double pores"; instead a strong signal is detected that forcedly needs to be attributed to the Eu ( $^{3+}$ cations (pointed by white arrows). A closer observation of these "new units" is shown in figure 3b. A 3D thermal colour map where the presence of two "double pores" can be identified is shown in figure S10. In here, it can be detected that rather than the faint signal, typical of these defects, a stronger contrast is obtained identifiyng three new different atomic positions, denoted as 1, 2 and 3 in the so-called double pores ${ }^{[6 b]}$. As it has been previously mentioned STEM-HAADF mode of operation is directly related to the atomic number of the elements and it is therefore the most advantageous approach for metal loaded supports. The relatively high contrast difference (in HAADF mode) between these defects and the rest of the framework can be easily understood in terms of $Z$ contrast. At those specific positions the signal coming from the framework is virtually non-existent, as the scattering phenomena is strongly affected by the atomic number; heavy elements scatter more electrons making them very bright in comparison with the $\mathrm{Si}$, $\mathrm{Ti}$ and $\mathrm{O}$. A closer observation is presented in figure $3 c$; the $C_{s}$-corrected HAADF data displays the presence of a "double pore". For clarity, in the superimposed model (where Eu appears in purple) this defect is represented as a complete pore along the $z$ axis; however, in the experimental image situated underneath the model, a weak framework signal? can be observed. As described elsewhere ${ }^{[1 c]}$ this is due to the presence of another Ti unit separated by $6.7 \AA$ to the adjacent one. Figure $3 d$ shows a magnified image of two $\mathrm{Ti}$ chains that encapsulate the $\mathrm{TiO}_{6}$ octahedra directly linked and separated by $6.7 \AA$. For a more clear understanding of the contrast observed in the experimental data, the model of the Eu-ETS-10 is presented in figure S11. Figure S11a corresponds to the model where theoretically the double pore has been completely formed in the same manner as presented in figure 3c. As this is not the real case, figure S11b displays the model with the "double pore" but includes a fainter signal from the $\mathrm{TiO}_{6}$ octahedra with $\mathrm{SiO}_{4}$ units, which are present along the $c$ axis (perpendicular to the plane of observation). For clarity, these two units added do not have the Eu cations placed in them yet. The fact that these structures are more transparent corresponds to the less number of atoms along the $z$ axis, which make them invisible by TEM and weak signals by STEM. If now, the Eu cations are introduced at the same positions as originally described, substituting for the $\mathrm{Na}$ (I) and (II) sites, the contrast observed in the double pores can be understood. The three additional signals observed, numbered as 1, 2 and 3 corresponds to the $\mathrm{Eu}^{3+}$ at the center of the 7MR (number 1); Eu at the corners of the butterfly unit, close to the Si columns (number 2) and again another Eu atomic column in the center of adjacent $7 \mathrm{MR}$. Further evaluation was performed by chemical analyses, Figure S12 represents the EDS profiles of Eu ${ }^{3+}, \mathrm{Tb}^{3+}$ and Gd ${ }^{3+} \mathrm{ETS}_{-}$ 10 , where $\mathrm{Tb}^{3+}$ and $\mathrm{Gd}^{3+}$ produce the same results as $\mathrm{Eu}^{3+}$ confirming the reproducibility of the ion exchange and of cationic location. The EDS analyses corroborate the metal content and also the remaining $\mathrm{K}^{+}$cations that have not been completely removed. However, no $\mathrm{Na}^{+}$signal can be observed, which proves that $\mathrm{Na}^{+}$cations can be fully exchanged.

In conclusion, ETS-10 in its sodium and potassium form has been synthesized via hydrothermal methods. These cationic species compensate the two negative charges that each $\mathrm{TiO}_{6}{ }^{2-}$ unit introduces into the framework. In the present study, the power of spherical aberration STEM has been demonstrated as it has been able to locate the cationic sites that have been previously ascribed to be $\mathrm{Na}^{+}$positions. By the analysis of the atomic resolution images it has been possible to locate for the first time light elements within a porous network.

With the intention of producing materials with luminescent properties, three different rare earth metals were ionexchanged into ETS-10. The powered XRD analysis did not suggest any significant structural changes but it indicates that the trivalent metals went inside the pores replacing the initial alkali cations. The $\mathrm{C}_{\mathrm{s}}$-corrected STEM analysis made able to locate the positions of the $\mathrm{Eu}^{3+}$ cations in the ETS-10 framework and helped to understand the initial $\mathrm{Na}^{+}$conformation. The results provided here provides unambiguous evidences of the cationic positions in ETS-10 porous framework as well as proof the paramount importance of this technique for the analysis of beam sensitive materials doped with active species which is the key for the incoming new materials with improved catalytic, optical and electronic properties.

\section{Acknowledgements}

The research leading to these results has received funding from the European Union Seventh Framework Programme under Grant Agreement 312483 - ESTEEM2 (Integrated Infrastructure Initiative-13). 
[1] (a)M. Tamarasso, G. Perego, B. Notari, U. S. Patent No. 4410501, 1983; (b)M. W. Anderson, O. Terasaki, T. Oshuna, A. Philippou, S. P. Mackay, A. Ferreira, J. Rocha, S. Lidin, Nature 1994, 367, 347-367; (c)A. Mayoral, J. Coronas, C. Casado, C. Tellez, I. Díaz, Chem. Cat. Chem. 2013, 5, $2595-2598$.

[2] (a)S. Uma, S. Rodrigues, I. N. Martyanov, K. J. Klabunde, Micropor. Mesopor. Mater. 2004, 67, 181-187; (b)Z. Lin, J. Rocha, A. Navajas, C. Tellez, J. Coronas, J. Santamaria, Micropor. Mesopor. Mater. 2004, 67, 79-86.

[3] X. Wang, A. J. Jacobson, Chem. Commun. 1999, 973-974.

[4] C. Casado, Z. Amghouz, J. R. Garcia, K. Boulahya, J. M. Gonzalez-Calbet, C. Tellez, J. Coronas, MRS Bulletin 2009, 44, $1225-1231$.

[5] (a)M. W. Anderson, J. R. Agger, D. P. Luigi, A. K. Baggaley, J. Rocha, Phys. Chem. Chem. Phys. 1999, 1, 2287-2292; (b)M. Grillo, J. Carrazza, J. Phys. Chem. 1996, 100, 12261-12264; (c)A. Damin, F. X. L. Xamena, B. Civalleri, C. M. Zicovich-Wilson, A. Zecchina, J. Phys. chem. B 2004, 108, 1328-1336; (d)W. Y. Ching, Y.-N. Xu, Z.-Q. Gu, Phys. Rev. B 1996, 54, R15585-R15589; (e)M. Koç, S. Galioglu, D. Toffoli, H. Ustunel, B. Akata, J. Phys. Chem. C 2014, 118, $27281-27291$.

[6] (a)I. Díaz, A. Mayoral, Micron 2011, 42, 512-527; (b)A. Mayoral, P. A. Anderson, I. Diaz, Micron 2015, 68, 146-151; (c)A. Mayoral, T. Carey, P. A. Anderson, A. Lubk, I. Diaz, Angew. Chem. Int. Ed. 2011, 50, 11230-11233.

[7] A. Mayoral, R. Arenal, V. Gascon, C. Marquez-Alvarez, R. M. Blanco, I. Diaz, Chem. Cat. Chem 2013, 5, 903-909.

[8] A. Mayoral, L. F. Allard, D. Ferrer, R. Esparza, M. Jose-Yacaman, J. Mater. Chem. 2010, 21, 893-898.

[9] C. Lamberti, Micropor. Mesopor. Mater. 1999, 30, 155-163.

[10] Z. Lin, J. P. Rainho, J. Domingues, L. D. Carlos, J. Rocha, Micropor. Mesopor. Mater. 2005, 79, 13-19.

[11] (a)A. Mayoral, S. Mejia-Rosales, M. M. Mariscal, E. Perez-Tijerina, M. Jose-Yacaman, Nanoscale 2010, 2, 2647-2651; (b)A. Mayoral, M. Sanchez-Sanchez, A. Alfayate, J. Perez-Pariente, I. Diaz, Chem. Cat. Chem. 2015, 7, 3719-3724. 

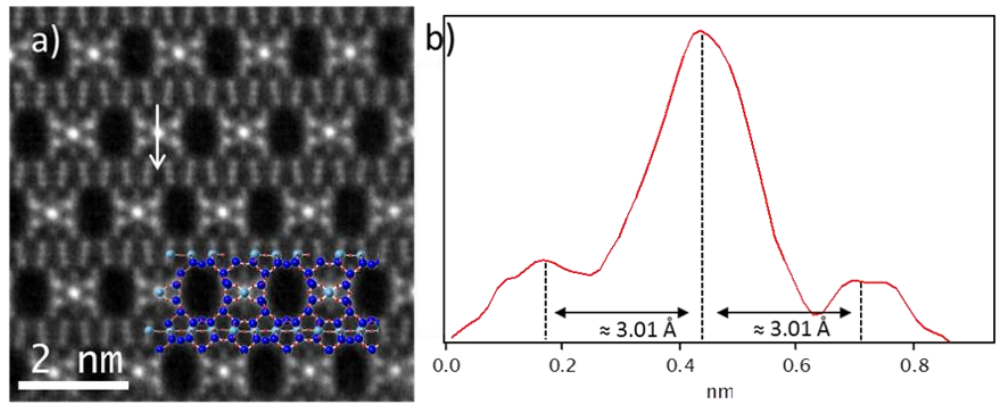

c)

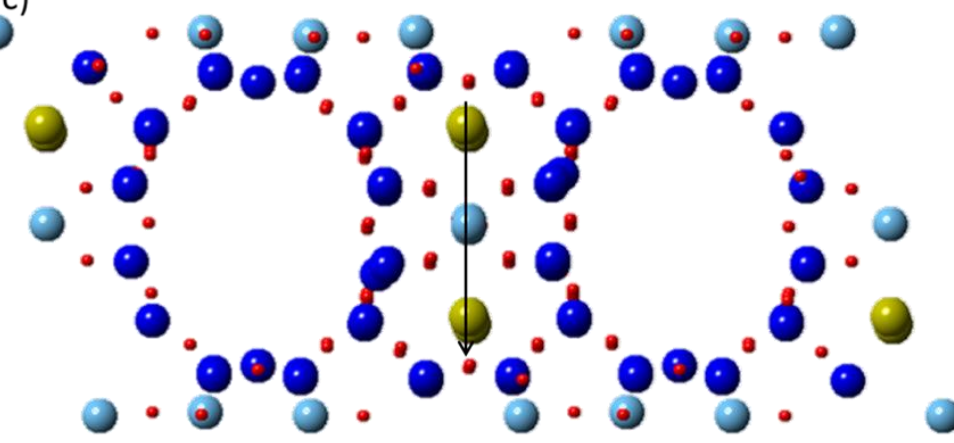

Figure 1. a) Atomic-resolution $C_{s}$-corrected STEM-HAADF image of ETS-10 on the [110]. The model with same colour code as in figure 1 is shown inset superimposed. The white arrow marks where the intensity profile was extracted from. b) Intensity profile. c) Ball model of ETS-10, where some possible Na+ sites are represents by yellow spheres. The black arrow corresponds to the same experimental positions as those in figure 2a. Color code: Dark blue $=\mathrm{Ti}$; light blue $=\mathrm{Si}$; red $=$ oxygen and yellow $=\mathrm{Na}$. 

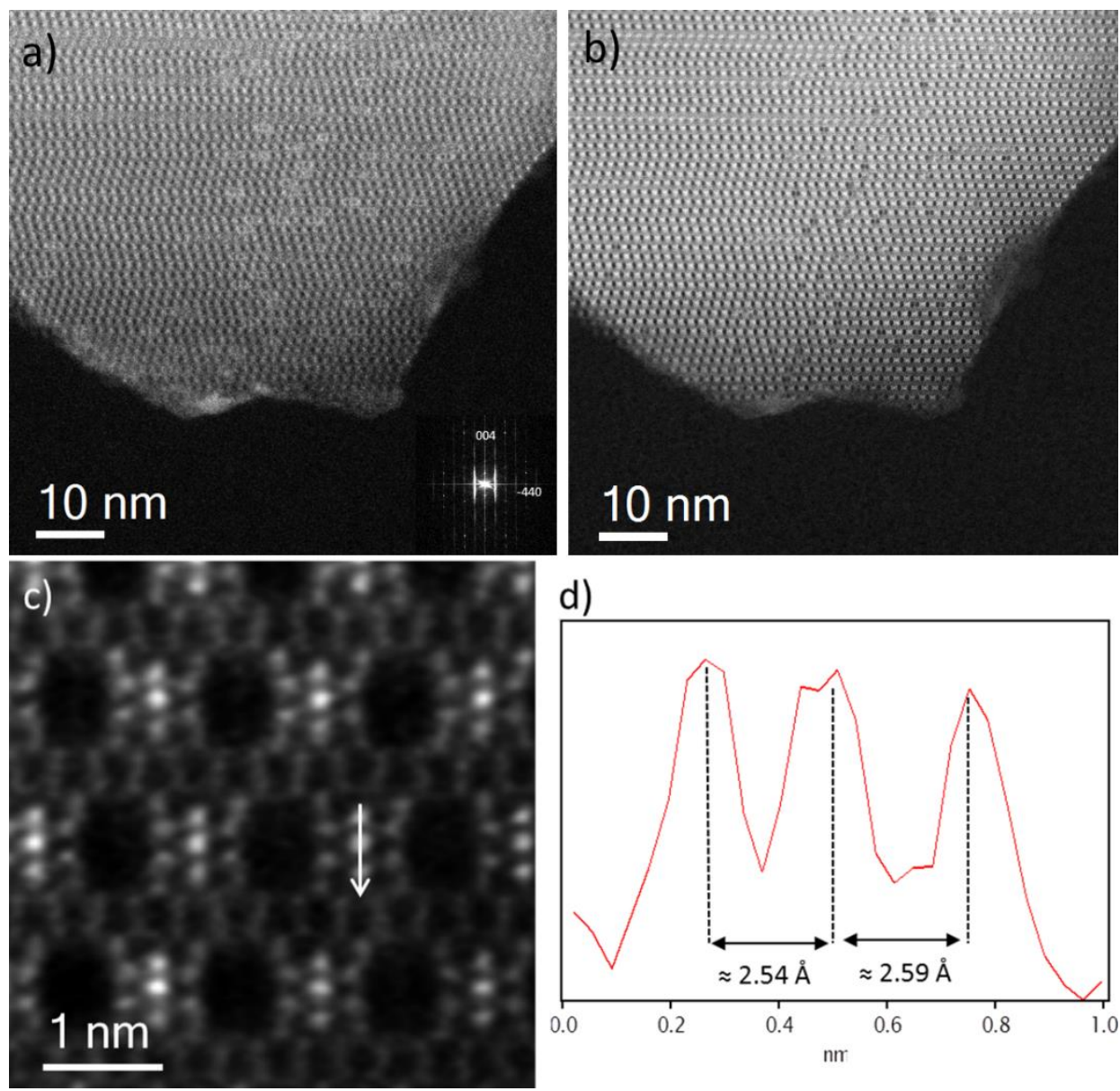

Figure 2. a) $\mathrm{C}_{\mathrm{s}}$-corrected STEM-HAADF of Eu-ETS-10 with the FFT shown inset and indexed assuming $\mathrm{C} 2 / \mathrm{m}$.. b) Same image collected with an annular dark field ADF detector. $\mathrm{c}$ Atomic resolution image observing all atomic columns conforming the material. d) Intensity profiles extracted from the white arrow plotted in figure $c$. 

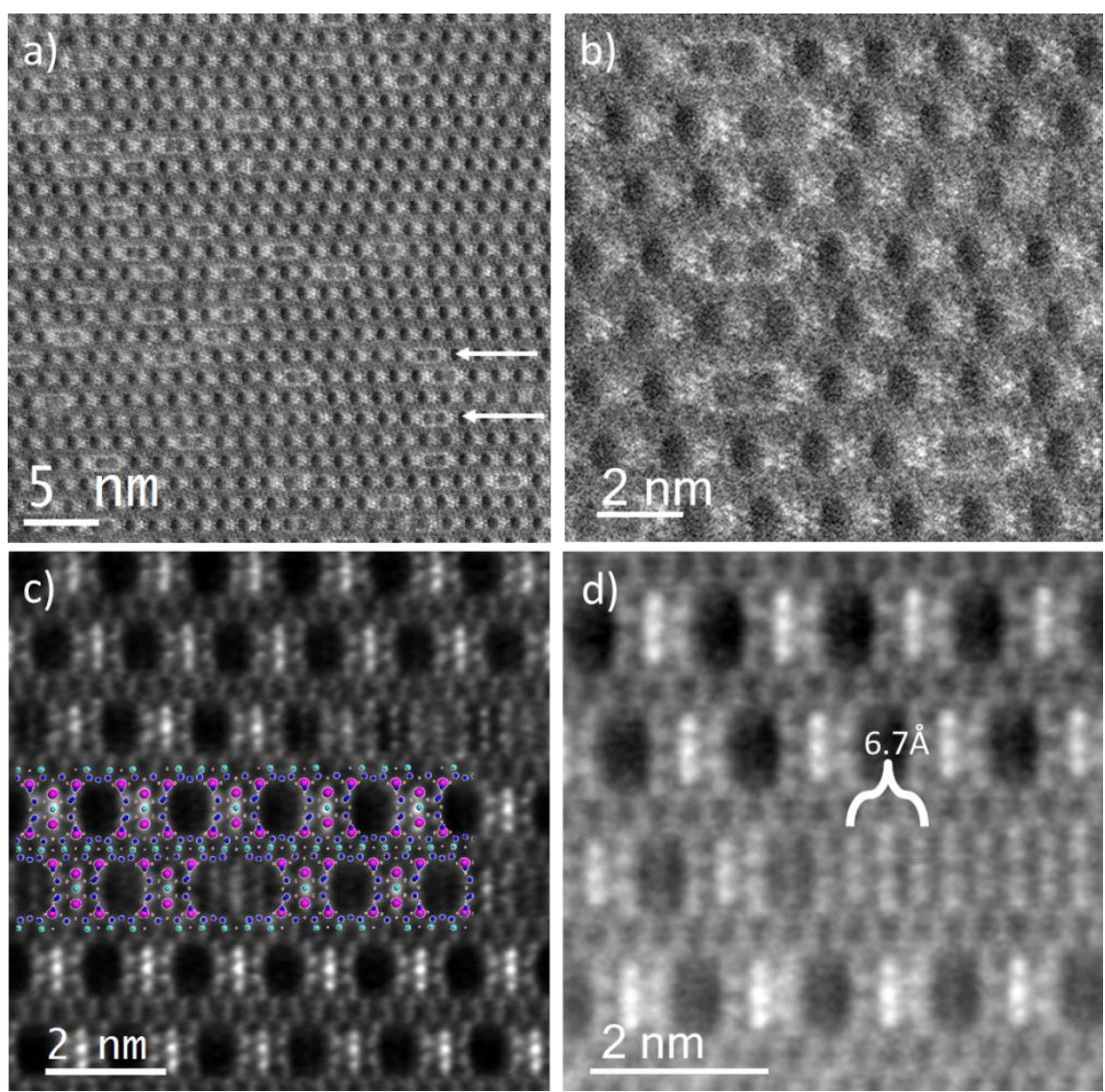

Figure 3. a) $\mathrm{C}_{\mathrm{s}}$-corrected STEM-HAADF of Eu-ETS-10, where a new different and stronger contrast can be identified pointed by white arrows. b) Closer observation of the area where this new defects are present. c) Atomic resolution image where the "double pores" are present. The ETS-10 model, with Eu ${ }^{3+}$ in purple, is superimposed. d) A closer observation of the defects with two Ti units are separated by approximately $6.7 \AA$. 